\title{
Prevalence of Medical Conditions among Dental Patients at a Community-based Dental Clinic, Trinidad: A Preliminary Investigation
}

\author{
${ }^{1}$ Ramaa Balkaran, ${ }^{2}$ Avind Harracksingh, ${ }^{3}$ Nicoli Rajcoomar, ${ }^{4}$ Keiron Jackson, ${ }^{5}$ Sarah Deosaran, ${ }^{6}$ Nadeline Gaffoor \\ ${ }^{7}$ Diana Haripersaud, ${ }^{8}$ Racquel Lutchmedial, ${ }^{9}$ Amy Maharaj, ${ }^{10}$ Amit Ramharacksingh, ${ }^{11}$ Vanesha Sewalia
}

\begin{abstract}
Objective: To describe the prevalence of medication used by patients of a community-based dental clinic and the prevalence of their various medical conditions.
\end{abstract}

Materials and methods: Hundred patients attending a community-based dental clinic were invited to participate in this study. Data were collected via a self-administered questionnaire which was distributed to a convenience sample. Descriptive data, such as age, gender, medical conditions, and medication used were recorded. The patients' medical conditions were then classified into general groups and analyzed using Statistical Package for the Social Sciences (SPSS) version 22.

Results: The participants had a mean age of 42 . The majority $(58 \%)$ were female. Most $(49 \%)$ participants were Afro-Trinidadians. The vast majority $(83 \%)$ did not have a dentist. Some (17\%) participants had been hospitalized for either illness and/or surgery in the past. Most (56\%) participants were not taking any form of medication, while $44 \%$ of participants displayed a wide range of medication use. Some $(30 \%)$ had only one medical condition present, and $14 \%$ were on a combination of medical drugs, in order to treat multiple medical conditions. The medical conditions with the highest prevalence were hypertension (14\%), diabetes $(11 \%)$, and ophthalmic disease (7\%).

Conclusion: These findings emphasize the array of medical conditions which must be taken into consideration in the dental setting as systemic health issues and their associated medications play an important role in treatment planning.

Keywords: Dental patients, Medical conditions, Medication usage.

How to cite this article: Balkaran $R$, Harracksingh $A$, Rajcoomar N, Jackson K, Deosaran S, Gaffoor N, Haripersaud D, Lutchmedial R, Maharaj A, Ramharacksingh A, Sewalia V. Prevalence of Medical Conditions among Dental Patients at a Community-based Dental Clinic, Trinidad: A Preliminary Investigation. J Oral Health Comm Dent 2018;12(2):51-55.

\footnotetext{
${ }^{1}$ Lecturer, ${ }^{2}$ Part-time Lecturer, ${ }^{3-11}$ Student

${ }^{1-11}$ School of Dentistry, The University of the West Indies, Champs Fleurs, Trinidad and Tobago

Corresponding Author: Ramaa Balkaran, Lecturer, School of Dentistry, The University of the West Indies, Champs Fleurs Trinidad and Tobago, Phone: +18684977848 , e-mail: ramaa. balkaran@gmail.com
}

\section{Source of support: Nil}

Conflict of interest: None

\section{INTRODUCTION}

Dental patients are susceptible to systemic illnesses in addition to diseases within the oral cavity. It has also been suggested that the oral cavity can show early signs of systemic diseases, for instance, periodontal disease has been associated with systemic diseases, such as diabetes, hypertension, and pulmonary disease. Individuals are usually prescribed various medications to manage and treat their systemic ailments. Oral health care providers must pay particular attention to medication history as well as patient history as various complications can result from these medications, such as xerostomia and candidiasis. Studies have categorized medically compromised patients' conditions into these conditions: Allergies, endocrine disorders, cardiovascular disorders, respiratory disease, hematological disorders, liver disease, renal disease, and others. ${ }^{1,2}$ Trinidad and Tobago is a multicultural and multiracial society that is burdened by the aforementioned conditions, as well as infectious diseases. The population trends exhibit an aging population. This trend may be due to cutting-edge technologies and accessibility to health procedures and management or the possibility of the public being educated on the benefits of self-care and proper hygiene. Moreover, the availability of approved and tested medications and supplements has accounted for such a phenomenon. People are more likely to visit dentists, as they become older and it is important that dentists are aware of the possible complications that can occur when treating patients with certain medical conditions or who are taking certain medications. ${ }^{2}$

Cardiovascular medications have been shown to be the most prevalent drugs used followed by endocrinologic drugs, nutritional therapeutics, and drugs acting on the musculoskeletal system. ${ }^{2}$ According to the World Health Organization (WHO), the most common oral diseases are dental caries, periodontal diseases, and oral cancers. ${ }^{3}$ Dental patients are more likely to visit a dentist in times of oral distress and pain, and may present to 
the dentist with possible oral complications. Many oral medications contain a sugar-coated layer and therefore, increase the likelihood of carious lesions and further oral complications of medications. Previous studies have also shown that there is a correlation between the use of antiasthmatic medication and dental caries, particularly in children. ${ }^{4}$ The role of the oral health provider is to ensure proper management of oral health and hygiene by the patient; this includes the education of the possible side effects of the medication usage on oral health to the patient. This study seeks to describe the classification of medication used and the general health status among dental patients attending a community-based dental clinic in Trinidad.

\section{MATERIALS AND METHODS}

One hundred patients attending the University of the West Indies (UWI) Arima Dental Clinic were invited to participate in this study, over a period of 6 months. A self-administered questionnaire was piloted on patients at the main clinic at the UWI School of Dentistry for content and ease of use. It was then distributed to a convenience sample of dental patients at the Arima Dental Clinic. The patients' various medical problems and medication use were recorded and categorized into general groups: Cardiovascular/respiratory, hematological, immune system/infectious disease, endocrine/ digestion, gastrointestinal/urinary, and neurological/ muscular Skeletal. The classification of the medications can be found in Table 1 .

The items in the questionnaire were closed. Descriptive statistics were recorded for the various ages, gender, and related to their medical conditions and medication used. The data were analyzed using SPSS version 22 . The study was approved by the Campus Ethics Committee, UWI, St. Augustine.

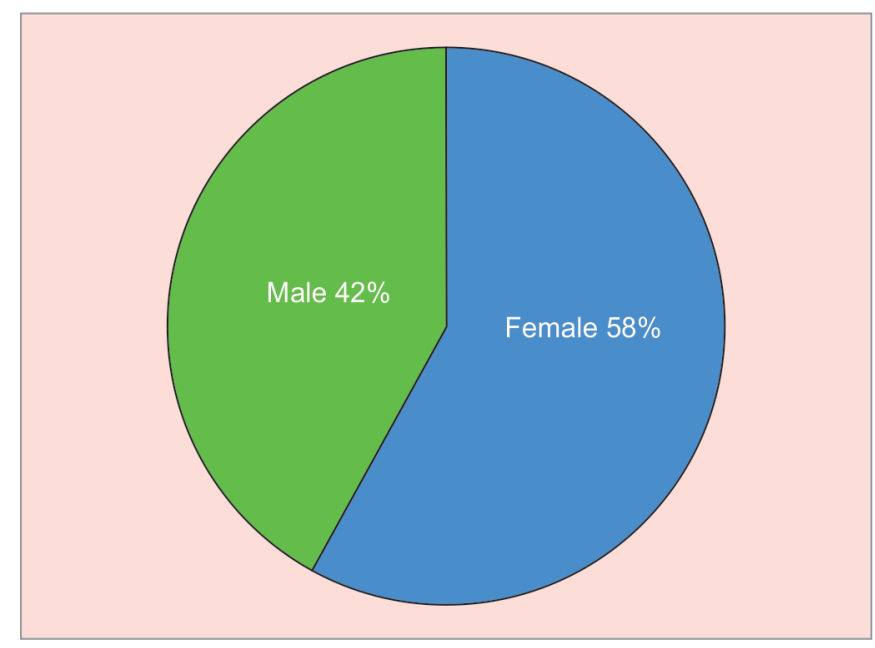

Graph 1: Distribution of male and female participants in the study
Table 1: Names of medications given by patients and classification

\begin{tabular}{ll}
\hline Medications used & Classification \\
\hline Diamicron, Metformin, Insulin, Diaban & Antihyperglycemic \\
Paracetamol, Tramacet ${ }^{\circledR}$, Tegretol, & Analgesic \\
Panadol, Aspirin, Flamar ${ }^{\circledR}$, NSAID, &
\end{tabular}

Ponstan Forte ${ }^{\circledR}$, Motrin, Olfen, Tegretol

Amoxicillin, Flagyl, Diaflex, Amoxil Antibiotic

Enalapril, Nifedipine, Nifelat, Methyldopa, Antihypertensive Vasopril, Bendrofluazide Hydralazine,

Atenolol

Evening Primrose, Tonic, SkinHairNails ${ }^{\circledR}$, Supplement Women's One a Day ${ }^{\circledR}$, Vitamin C, Iron Supplement, Ginkgo Biloba, Omega $\mathrm{XL}^{\circledR}$ Plavix $^{\circledR}$, GTN, Coreg, Simlo, Simvastatin Amitriptytine

Flomax

Omeprazole, Lomotil

Antipsychotic

Prostate

Steroid Inhaler

Gastric

Respiratory

NSAID: Nonsteroidal anti-inflammatory drugs; GTN: Glyceryl trinitrate

\section{RESULTS}

The participants ranged between the ages of 16 and 87 years, with a mean age of 42 years. The female participants comprised 58\% (Graph 1); 49\% of the participants identified as Afro-Trinidadians and $21 \%$ of participants identified as Indo-Trinidadians (Graph 2).

\section{Dental Attendance}

Of the participants, $17 \%$ had a dentist while the remaining $83 \%$ of participants did not. Of the patients who had dentists, $11 \%$ had last visited within 6 months, $10 \%$ had last visited approximately within a year, 55\% had last visited over a year ago, and $24 \%$ of participants never visited a dentist and/or dental clinic; $67 \%$ of participants only visited their dentist when there was a dental emergency, $11 \%$ participants had never visited a dentist prior to the visit at the time of data collection, $2 \%$ participants

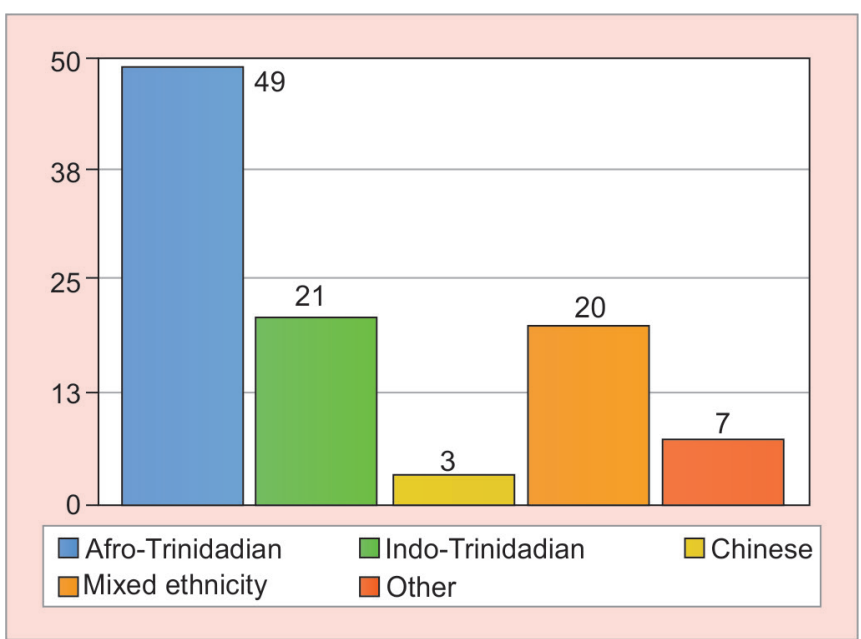

Graph 2: Ethnic distribution among the participants 
visited once per year, $14 \%$ participants visited more than once per year, and the remaining $6 \%$ patients visited their dentist every few years.

\section{General Health}

Changes in the past year were experienced by $7 \%$ of the participants. It was noted that $17 \%$ of the participants had been hospitalized for either illness and/or surgery in the past. Additionally, $12 \%$ of participants were advised to take antibiotics before dental treatments. It was observed that $5 \%$ of participants previously had an adverse reaction to medications and or injections, and $10 \%$ of participants suffered from allergies. Of the $58 \%$ of women studied, $3 \%$ were pregnant at the time of data collection.

\section{Prevalence of Medicines}

Of the participants, 56\% were not taking any form of medical drugs or supplements, while $44 \%$ of participants displayed a wide range of medical drug use relevant to their medical conditions (Table 2); 30\% of participants used medication in relation to only one medical condition present, such as analgesics (13\%); antihyperglycemics (5\%); antihypertensives (5\%); supplements (4\%); stomach acid-reducing medicines (1\%); antipsychotics (1\%); and benign prostatic hyperplasia (1\%). Furthermore, $14 \%$ of participants studied were on a combination of medical drugs in order to treat more than one medical condition, such as (in descending order): Antihyperglycemic and antihypertensive (3\%); cardiac and antihypertensive (2\%); analgesic and supplements (2\%); analgesic and antibiotic (2\%); antihyperglycemic diabetic, cardiac, and

Table 2: Medications used by patients at the UWI Arima Dental Clinic

\begin{tabular}{ll}
\hline & Total \\
Drug class and combinations & $5(5 \%)$ \\
\hline Antihyperglycemics & $13(13 \%)$ \\
Analgesics & $4(4 \%)$ \\
Supplements & $1(1 \%)$ \\
Gastric medication & $1(1 \%)$ \\
Antipsychotics & $5(5 \%)$ \\
Antihypertensives & $3(3 \%)$ \\
Antihyperglycemics and antihypertensives & $1(1 \%)$ \\
Antihyperglycemics and supplements & $2(2 \%)$ \\
Cardiac and antihypertensives & $2(2 \%)$ \\
Analgesics and supplement & $2(2 \%)$ \\
Analgesics and antibiotics & $1(1 \%)$ \\
Respiratory and analgesics & $1(1 \%)$ \\
Cardiac and antibiotics & $1(1 \%)$ \\
Benign prostatic hyperplasia-reducing medicines & $1(1 \%)$ \\
Antihyperglycemic, cardiac and antihypertensives & $1(1 \%)$ \\
Analgesics, antihypertensives and antihyperglycemics & $56(56 \%)$ \\
No medication &
\end{tabular}

antihypertensive (1\%); respiratory and analgesic (1\%); antihyperglycemic and supplements (1\%); cardiac and antibiotic (1\%); and analgesic, antihypertensive, and antihyperglycemic (1\%).

The three conditions with the highest prevalence were hypertension (14\%), followed by diabetes (11\%), and then ophthalmic disorders $(7 \%) ; 6 \%$ of participants experienced problems with their sinuses, $4 \%$ of participants experienced shortness of breath, asthma, acid reflux and were tobacco users, $3 \%$ of participants experienced chest pain, and $2 \%$ of the sample population experienced angina, heart disease, swollen ankles, and had blood disorders. The minority of the sample population (1\%) experienced myocardial infarction, heart murmurs, stroke, abnormal bruising, arthritis, had human immunodeficiency virus / acquired immune deficiency syndrome, another form of sexually transmitted diseases, and dietary restrictions.

\section{DISCUSSION}

The participants who attended the community-based dental clinic shared a similar demographic trend to the population in the borough of Arima ${ }^{5}$ and any minor differences observed may have been due to individuals attending dentists in the private sector or those who did not visit a dentist at all, since our study showed that $83 \%$ of the participants did not have a dentist.

Demographic statistics are important, as in many systemic diseases there are observable trends according to various demographic information, such as age, sex, and ethnicity. Furthermore, ethnicity also has a role in culture and in particular, ethnic foods, carbohydrate-rich food and drink also account for the prevalence of diseases, such as diabetes mellitus, hypertension, and cardiac disease. In addition, diabetic patients are at an increased risk of periodontal disease. ${ }^{6}$

Dentistry is associated with anxiety by patients for even the simplest of procedures. ${ }^{7}$ As such, most dental patients tend to only visit dentists during what they may consider a dental emergency or when there is an onset of pain. This is evident in our study where only $17 \%$ of the sample had dentists, and only $21 \%$ of that visited a dentist within one year of participating in the survey. This is further emphasized in this study where $67 \%$ of the patients with dentists only visited their dentist during dental emergencies. The fear of regular dental visits can account for the high prevalence of oral issues found in Trinidad and Tobago. In 2000, diabetes mellitus, malignant neoplasm, and cerebrovascular disease accounted for $67 \%$ of all deaths in the country. ${ }^{8}$ Nevertheless, according to the $\mathrm{WHO}$, the number one cause of death in 2000 was heart disease which includes ischemic heart disease 
and hypertensive heart disease. ${ }^{3}$ It was observed that of the $44 \%$ of the sample population that uses medication, the majority catered for diabetes mellitus, cardiac disease, and hypertension (19\%).

Analgesics were the most common drug used by the patients $(13 \%)$. This is possibly due to the fact that any distress or discomfort in the oral cavity will always result in some sort of pain experienced by that patient. Furthermore, as aforementioned, most patients tend to wait only until there are issues experienced or what they consider dental emergencies before they decide to visit a dentist. The consumption of analgesics may be a method of delaying dental visits during any distress in the oral cavity. The results showed the trend that the majority of participants did not suffer any ailments and only a minority of the sample experienced several medical conditions.

In our study, 5\% used antihypertensive medications compared with a Canadian-based study ${ }^{9}$ in which it was found that approximately a third of patients in a periodontal private practice were taking an antihypertensive medication.

The majority of the population, $56 \%$, was not taking any medication, which may have been due to their mean age being 42 , in that most patients in this age group do not use medications compared with the elderly population, patients 65 years and older, who usually have more complex medical conditions. ${ }^{10}$ Additionally, the majority of patients, in this study were not diagnosed with medical problems which warranted medication.

In our study, only $5 \%$ of the patients were on medications for diabetes, which was similar in prevalence to a local study in which $6 \%$ reported having diabetes. ${ }^{11}$ It is still vital that the dental practitioner is aware of the diabetes status and glycemic control of the patient, given that there are many well-established associations among diabetes and periodontal disease where $67.2 \%$ of adults with diabetes in Trinidad had moderate or advanced periodontal disease. $^{6}$

\section{Limitations}

The patients may not have reflected their true opinions and their answers to the questionnaire may have been subjected to recall bias in the medication list. This was a preliminary study and the sample size was small, and therefore, only descriptive statistics were performed. Additionally, given that this was a convenience sample, the results may not represent the general population.

\section{Recommendations}

A detailed medical history, social history, and a list of medications used by the patient are the tenets of good dental treatment. Dental patients can experience an array of medical conditions in which their health status may change between visits to the dentist; in addition, many systemic conditions have specific oral manifestations. This information will therefore, aid in diagnosis, direct the dentist to which procedures should be done as well as which medications can be prescribed without causing negative drug interactions. Future research could involve the collection of clinical data and then correlate the dental findings with systemic diseases and medications. Additionally, governmental procurement agencies may use this information to aid with medication provision to ensure their availability at public health centers.

\section{CONCLUSION}

This study highlighted some of the more prevalent systemic illnesses seen in dental patients and the common medications used by the patient. It underscores the importance of a well-recorded and detailed patient history, thereby providing the most suitable diagnosis, treatment, management, and prognosis of oral diseases experienced in dental patients. This is essential, given that certain dental procedures may be contraindicated or require modification based upon the patients' health status and medications. In addition, certain systemic conditions have specific oral manifestations and the dentist may be the first medical professional to note these changes in many cases.

\section{ACKNOWLEDGMENTS}

The authors would like to thank the staff and patients of the UWI Arima Dental Clinic for their assistance and participation in this study. They wish to thank Dr Haytham Al-Bayaty for his kind assistance with this research.

\section{REFERENCES}

1. Dhanuthai K, SappayatosokK, Bijaphala P,KulvititS, Sereerat T. Prevalence of medically compromised conditions in dental patients. Med Oral Pathol Oral Cir Bucal 2009 Jun;14(6): E287-E291.

2. Jainkitvtivong A, Aneksuk V, Langlais RP. Medical health and medication use in elderly dental patients. J Contemp Dent Pract 2004 Feb;5(1):31-41.

3. World Health Organization (WHO). Oral Health. http:// www.who.int/mediacentre/factsheets/fs318/en/. Accessed: February 12, 2016.

4. Shashikiran ND, Reddy VV, Raju PK. Effect of anti-asthmatic medication on dental disease: dental caries and periodontal disease. J Indian Soc Pedod Prev Dent 2007 Apr-Jun;25(2): 65-68. 
5. Central Statistical Office of the Government of Trinidad and Tobago (CSO). Trinidad \& Tobago 2011 Demographic Report. http:/ /cso.gov.tt/default/publicationcontainer/ trinidad-tobago-2011-demographic-report/. Accessed: June 07, 2016.

6. Balkaran R, Naidu R, Teelucksingh S, Seemungal T, Pinto Pereira L, Prayman E, Bissoon A. A preliminary investigation of periodontal disease and diabetes in Trinidad. West Indian Med J 2011 Jan;60(1):86.

7. Naidu RS; Lalwah S. Dental anxiety in a sample of West Indian adults. West Indian Med J 2010 Oct;59(5):567-572.

8. World Health Organization (WHO). Country cooperation strategy: Trinidad and tobago. http://www.who.int/ countryfocus/cooperation_strategy/ccs_tto_en.pdf?ua=1. Accessed: June 07, 2016.

9. Fitzgerald J, Epstein JB, Donaldson M, Schwartz G, Jones C, Fung K. Outpatient medication use and implications for dental care: Guidance for contemporary dental practice. J Can Dent Assoc 2015;81:f10.

10. Aggarwal A, Panat SR, Talukder S. Self-reported medical problems among dental patients in western Uttar Pradesh, India. J Dent Educ 2011;75(12):1635-1640.

11. Al-Bayaty HF, Murti PR, Naidu RS, Matthews R, Simeon D. Medical problems among dental patients at the School of Dentistry, The University of the West Indies. J Dent Educ 2009 Dec;73(12):1408-1414. 